\title{
Kawasaki Disease and Hypertension in an Infant
}

\author{
Shirin Sayyahfar, ${ }^{1, *}$ and Rozita Hoseini ${ }^{2}$ \\ ${ }^{1}$ Assistant Professor, Subspecialist of Pediatric Infectious Diseases, Department of Pediatrics, Ali Asghar Children Hospital , Iran University of Medical Sciences, Tehran, Iran \\ ${ }^{2}$ Assistant Professor, Pediatric Nephrologist, Department of Pediatrics, Ali Asghar Children Hospital, Iran University of Medical Sciences, Tehran, Iran \\ "Corresponding author: Shirin Sayyahfar, MD, Department of Pediatrics, Iran University of Medical Sciences, Ali Asghar Children Hospital, Vahid Dastgerdi St., Shariati St., P. O. \\ Box: 1919816766, Tehran, Iran. Tel: +98-2122255218, Fax: +98-21220063, E-mail: sayyahfar.sh@iums.ac.ir
}

Received 2016 May 06; Revised 2016 September 20; Accepted 2016 October 17.

\begin{abstract}
Introduction: Kawasaki disease (KD) is the second most common vasculitis of medium-sized vessels in children. Hypertension is rarely reported in this disease.

Case Presentation: Herein, we report a 5-month-old male infant with atypical KD and new onset of hypertension without cardiac involvement or renal vessel stenosis. Hypertension was self-limited and resolved within six months after the onset of disease.

Conclusions: Clinicians should consider KD as a potential source of secondary hypertension in children, and high blood pressure ought to be considered as a significant complication in Kawasaki patients.
\end{abstract}

Keywords: BCG Scar, Hypertension, Kawasaki Disease, Renal Disease, Vasculitis

\section{Introduction}

Kawasaki disease (KD) is the second most common vasculitis of medium-sized vessels in children (1). Coronary artery involvement is not the sole important complication of this disease, but renal vessels could also suffer during the course of this disease (2). Renal vasculitis may occur during the progression of this disease and cause transient hypertension, renal stenosis, and more pronged complications (3). Herein, we report a 5-month-old male infant with atypical KD and hypertension without any cardiac involvement or renal vessel stenosis. This study is a case report of an infant affected with KD in association with transient hypertension but no documented cardiac, coronary artery, or renal vessel abnormality, which is an unusual complication of this disease.

\section{Case Presentation}

A previously healthy 5-month-old male infant having symptoms such as fever $\left(39^{\circ} \mathrm{C}\right)$, restlessness, diarrhea, and macular rash for three days was referred to Ali Asghar Children's hospital, Tehran, Iran. On physical examination, he showed signs of bilateral nonexudative conjunctivitis, dried and cracked erythematous lips, perioral peeling, and macular rash. The rash appeared on the abdomen and eventually spread to the limbs and was more intense in the diaper area (Figure $1 \mathrm{~A}$ and B). The site of BCG inoculation was erythematous and indurated (Figure 2). He did not have any cervical lymphadenopathy but had erythema and swelling of the extremities. Brachial blood pressure (bBP) was checked multiple times and was 140/90 mmhg at the emergency department and during the first day of admission. His complete blood count (CBC), erythrocyte sedimentation rate (ESR), C-reactive protein (CRP) and urinalysis results are shown in Table 1 . The results of CSF examination and blood and urine culture were unremarkable. Other laboratory data were within normal range. He was treated with a single dose of IVIG $2 \mathrm{gr} / \mathrm{kg}$ and aspirin 80 $\mathrm{mg} / \mathrm{kg}$ in four divided doses as preliminary treatment for diagnosis of KD. His fever dropped after completion of IVIG infusion and the conjunctivitis, rash, and erythema at BCG inoculation site disappeared within 24 - 48 hours.

Restlessness improved dramatically after completion of IVIG infusion, but hypertension still persisted. His high blood pressure was first controlled with hydralazine, and then switched to amlodipine. ECG and two-dimensional echocardiography with echo-colour Doppler showed no coronary aneurysm or abnormal finding. DMSA scan of kidneys and ultrasonography of the urinary tract system, color Doppler ultrasound of renal arteries, and magnetic resonance angiography (MRA) of aorta and renal arteries were all normal. He had no past history of umbilical catheterization or blood exchange. Also there was not any history of essential hypertension in the family. Holter monitoring during amlodipine treatment showed blood pressure in normal range for his age. He was discharged with amlodipine and antithrombotic dose of aspirin. His parents self-discontinued anti-hypertensive therapy 20 days after discharge and did not cooperate for follow-up visits. He was visited six months after hospital discharge, and no hypertension or complication was found. 

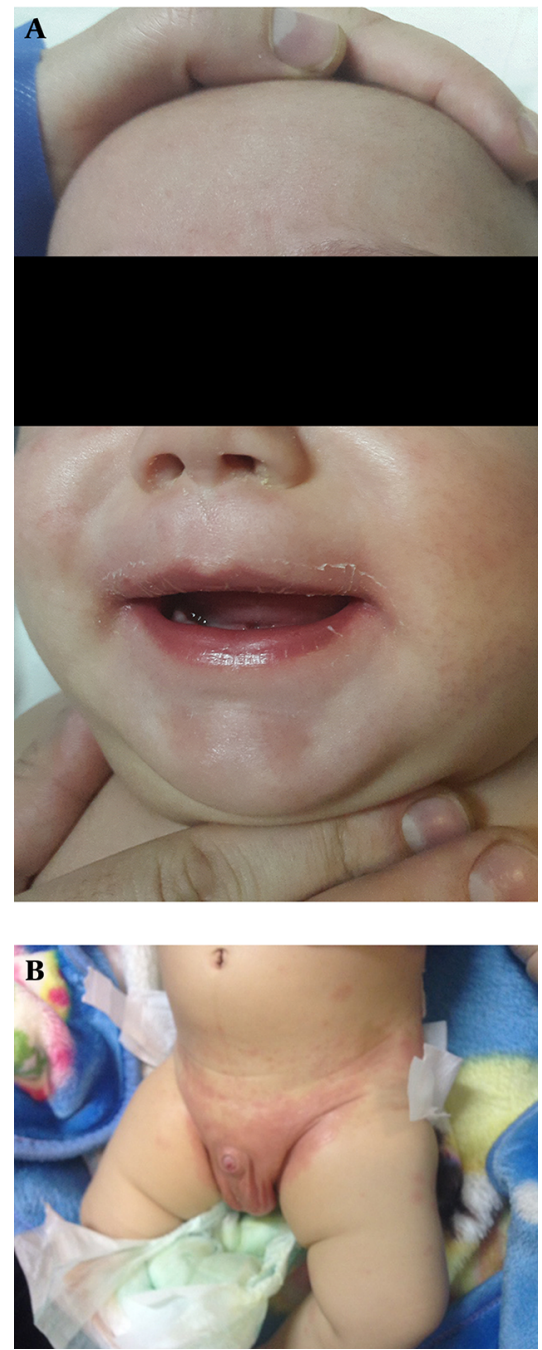

Figure 1. A, Dried and cracked erythematous lips with perioral peeling; B, intense macular rash, in the diaper area.

\section{Discussion}

For diagnosis of classic KD, patients should have fever for at least 5 days (or fever until the date of treatment if given before the fifth day of illness) and at least 4 of the 5 characteristic features without alternative explanation for the findings (4). Our patient had four specific features such as conjunctivitis, rash, erythema and swelling of the palms and soles, cracked lips but the duration of fever before treatment was less than 5 days. So this patient could be considered as classic case of KD. According to the american heart association (AHA) and american academy of pediatrics (AAP), "atypical" KD is stated if the patient with diagnosis of KD demonstrates atypical clinical and/or laboratory features (5). This patient had hypertension which

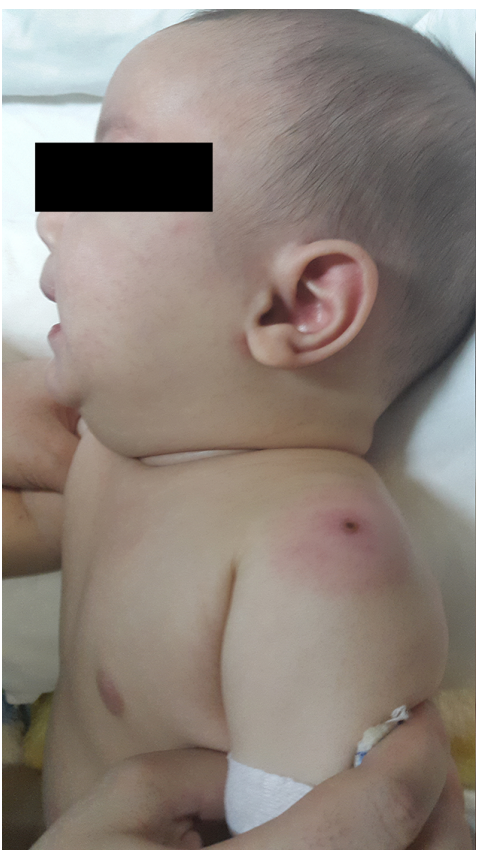

Figure 2. Erythema at the Site of BCG Inoculation

Table 1. Initial Blood Test Values

\begin{tabular}{|c|c|c|}
\hline Tests & Patient Value & Normal Value for the Age \\
\hline WBC $/ \mathbf{L}$ & $16.7 \times 10^{9}$ & $4-10 \times 10^{9}$ \\
\hline Neutrophil (\%) & 64 & $20-48$ \\
\hline Band (\%) & 12 & $<5$ \\
\hline Lymphocyte (\%) & 24 & $34-88$ \\
\hline $\mathrm{Hb}, \mathbf{g r} / \mathbf{d L}$ & 10.2 & $10.5-14$ \\
\hline Plt, $\mathbf{m m}^{3}$ & 378000 & $150000-450000$ \\
\hline ESR, $\mathbf{m m} / \mathbf{h}$ & 70 & $<15$ \\
\hline CRP, mg/L & 101 & $<10$ \\
\hline \multicolumn{3}{|l|}{ Urinalysis } \\
\hline Specific gravity & 1011 & $1005-1025$ \\
\hline $\mathrm{PH}$ & 6.5 & $4.5-8$ \\
\hline $\begin{array}{l}\text { WCB (per high } \\
\text { power field) }\end{array}$ & $20-22$ & $0-3$ \\
\hline $\begin{array}{l}\mathrm{RBC} \text { (per high power } \\
\text { field) }\end{array}$ & $8-10$ & $0-3$ \\
\hline Leukocyte esterase & $1+$ & negative \\
\hline Protein & $1+$ & $<150, \mathrm{mg} / \mathrm{dL}$ \\
\hline Blood & $1+$ & negative \\
\hline
\end{tabular}

Abbreviations: CRP, C-reactive protein; ESR, erythrocyte sedimentation rate; $\mathrm{Hb}$, hemoglobin; Plt, platelet; RBC, red blood cell; WBC, white blood cell. 
is not a usual presentation in $\mathrm{KD}$; therefore, our diagnosis was "classic atypical” KD.

Although the diagnosis of KD needs exclusion of the other differential diagnoses, erythema and induration at the site of BCG inoculation is a specific sign of this disease and a useful tool for early diagnosis of KD especially in countries with BCG vaccination at birth $(6,7)$. As a general rule and whenever $\mathrm{KD}$, even an incomplete type, is diagnosed, the appropriate treatment ought to be started because prolonged fever is the strongest risk factor for development of coronary artery aneurysms (8).

In view of the erythema at the site of the BCG vaccination, early diagnosis of KD led to our early treatment which may have caused the prevention of persistent fever $\geq 5$ days and appearance of other signs and symptoms of this disease.

Childhood hypertension should be evaluated and watched thoroughly because essential hypertension is rare in this age group (2). Renal diseases are the most common etiology of persistent hypertension in children (2). Renovascular disease accounts for $8-10 \%$ of all cases of paediatric hypertension, divided into extrinsic compression of renal arteries and intrinsic renal artery disease (2).

The etiology of hypertension in KD could be due to renal vasculitis leading to renal artery stenosis, or renal parenchyma involvement (3).

Although coronary artery vasculitis which may cause aneurysm is the most important complication of $\mathrm{KD}$, renal artery involvement leading to hypertension has been raised as a theoretical possibility of hypertension secondary to $\mathrm{KD}(2)$.

According to the routine health assessment during the first three months of life, and before the onset of the disease, the patient did not have hypertension. In addition the patient did not have hypertension 6 month after the disease while antihypertensive therapy was stopped .So he could not have essential hypertension. The echocardiography did not show any left ventricular hypertrophy, and the eye examination was normal in ophthalmoscopy. Therefore, it could be concluded that hypertension was acute and as a consequence of suffering from complications of KD.

Since the echocardiogram, color Doppler ultrasonography, and MRA of the renal arteries were normal, and the blood pressure reached the normal limit within six months even after discontinuation of the antihypertensive therapy, it seems that the most probable diagnosis was transient renal artery vasculitis.

Progression of the arterial lesions in KD is based on the duration of the illness. In the first stage of the disease ( 0 9 days), peri-vasculitis of small arteries is characterized (9). So early stage of KD vasculitis may have not been diagnosed by current imaging studies. This might be the reason why we could not find any abnormal finding in MRA and color Doppler ultrasound of renal arteries.

Although hypertension is not a usual complication associated with KD, we guess the real incidence of hypertension associated with this disease is underestimated. Usually the high blood pressure in infants and children suffering from this disease is considered the result of severe irritability and restlessness seen with this disease and overlooked by health care personnel. Since hypertension could be a transient or prolonged sequel of renal vasculitis, it is recommended to follow up the $\mathrm{KD}$ patients for probable hypertension, with at least taking blood pressure concurrent with follow-up echocardiograms, in order to screen for persistent hypertension. Whether or not erythema and induration at the site of the BCG vaccine scar is related to the severity of the disease and probable appearance of hypertension needs further evaluation.

\section{Conclusions}

In conclusion, clinicians should be aware in terms of monitoring and considering KD as a potential source of secondary hypertension in young infants and children. High blood pressure ought to be considered as a significant complication in Kawasaki patients.

\section{Footnotes}

Authors' Contribution: All authors contributed to the manuscript equally. Shirin Sayyahfar is the guarantor.

Funding/Support: None declared.

\section{References}

1. Falcini F, Calabri GB, Simonini G, Vitale A, De Simone L, De Martino M. Bilateral renal artery stenosis in Kawasaki disease: a report of two cases. Clin Exp Rheumatol. 2006;24(6):719-21. [PubMed: 17207393].

2. Foster BJ, Bernard C, Drummond KN. Kawasaki disease complicated by renal artery stenosis. Arch Dis Child. 2000;83(3):253-5. [PubMed: 10952649].

3. Watanabe T. Kidney and urinary tract involvement in kawasaki disease. Int J Pediatr. 2013;2013:831834. doi: 10.1155/2013/831834. [PubMed: 24288547].

4. American Academy of Pediatrics . Kawasaki Disease. In: Kimberlin DW, Brady MT, Jackson MA, Long SS, editors. Red Book: 2015 Report of the Committee on Infectious Diseases. 30 ed. Elk Grove Village, IL: American Academy of Pediatrics; 2015. pp. 494-500.

5. Newburger JW, Takahashi M, Gerber MA, Gewitz MH, Tani LY, Burns JC, et al. Diagnosis, treatment, and long-term management of Kawasaki disease: a statement for health professionals from the Committee on Rheumatic Fever, Endocarditis, and Kawasaki Disease, Council on Cardiovascular Disease in the Young, American Heart Association. Pediatrics. 2004;114(6):1708-33. doi: 10.1542/peds.2004-2182. [PubMed: 15574639]. 
6. Sinha R, Balakumar T. BCG reactivation: a useful diagnostic tool even for incomplete Kawasaki disease. Arch Dis Child. 2005;90(9):891. doi: 10.1136/adc.2004.071332. [PubMed:16113127].

7. Terp S, Bar-Meir M, Tan T. Inflammatory reaction at the site of a Bacille Calmette-Guerin vaccination scar in an adopted child. Clin Infect Dis. 2007;45(4):518-9. doi: 10.1086/520020. [PubMed: 17638200]
8. Cimaz R, Sundel R. Atypical and incomplete Kawasaki disease. Best Pract Res Clin Rheumatol. 2009;23(5):689-97. doi: 10.1016/j.berh.2009.08.010. [PubMed: 19853833].

9. Burns JC, Kushner HI, Bastian JF, Shike H, Shimizu C, Matsubara T, et al Kawasaki disease: A brief history. Pediatrics. 2000;106(2):27. [PubMed: 10920183]. 